\title{
MAXWELL-CHERN-SIMONS CASIMIR EFFECT
}

\author{
KIMBALL A. MILTON \\ Department of Physics and Astronomy, University of Oklahoma \\ Norman, OK 73019-0225, USA
}

\begin{abstract}
In odd-dimensional spaces, gauge invariance permits a Chern-Simons mass term for the gauge fields in addition to the usual Maxwell-Yang-Mills kinetic energy term. We study the Casimir effect in such a $(2+1)$-dimensional Abelian theory. For the case of parallel conducting lines the result is the same as for a scalar field. For the case of circular boundary conditions the results are completely different, with even the sign of the effect being opposite for Maxwell-Chern-Simons fields and scalar fields. We further examine the effect of finite temperature. The Casimir stress is found to be attractive at both low and high temperature. Possibilities of observing this effect in the laboratory are discussed.
\end{abstract}

\section{Introduction}

By now it is well-known that, for theories in odd-dimensional spaces, one can add a gauge invariant Chern-Simons mass term for the gauge field in addition to the usual Maxwell-Yang-Mills term. Recently there has been considerable interest in such a $(2+1)$-dimensional Abelian theory in connection with the studies of the fractional quantum Hall effect in semiconductors and of high- $T_{c}$ superconductivity in copper-oxide crystals. The Lagrangian for the Maxwell-Chern-Simons theory written in curvilinear coordinates is

$$
\mathcal{L}=-\sqrt{-g} \frac{1}{4} F^{\mu \nu} F_{\mu \nu}+\frac{1}{4} \mu \epsilon^{\mu \alpha \beta} F_{\alpha \beta} A_{\mu}
$$

where $g$ is the determinant of the metric $g_{\mu \nu}$ and $\epsilon^{\mu \alpha \beta}=\sqrt{-g} e^{\mu \alpha \beta}$ is a tensor density, with $\epsilon^{012}=1$. In terms of the dual tensor $F^{\lambda}=\frac{1}{2} e^{\lambda \alpha \beta} F_{\alpha \beta}$ we can rewrite (1) as

$$
\mathcal{L}=\frac{1}{2} \sqrt{-g}\left(F^{\lambda} F_{\lambda}+\mu F^{\lambda} A_{\lambda}\right) .
$$

Varying $\mathcal{L}$ with respect to $A_{\mu}$ we find the equations of motion

$$
\epsilon^{\mu \alpha \beta} \partial_{\alpha} F_{\beta}+\mu \sqrt{-g} F^{\mu}=0 .
$$

We solve these equations subject to perfect conductor boundary conditions. That is, the tangential electric field must vanish on the boundary, or the normal component of the dual field vanishes, $F_{n}=0$. It is interesting to note that this is precisely the condition necessary to ensure the gauge invariance of the Lagrangian (2). That is, the mass term in (2) is gauge invariant only if we neglect the surface term

$$
\frac{1}{2} \mu \int d x \sqrt{-g} F^{\lambda} \partial_{\lambda} \Lambda=\frac{1}{2} \mu \int d S_{\lambda} \sqrt{-g} F^{\lambda} \Lambda=0,
$$


which is true if the normal component of $F^{\lambda}$ vanishes on the bounding surfaces.

\section{Green's Functions}

The stress tensor density following from (2) is

$$
t^{\alpha \beta}=\sqrt{-g}\left(F^{\alpha} F^{\beta}-\frac{1}{2} g^{\alpha \beta} F_{\lambda} F^{\lambda}\right) .
$$

We introduce the Green's function $G_{\mu \nu}$ according to

$$
F_{\mu}(x)=\int d x^{\prime} \sqrt{-g\left(x^{\prime}\right)} G_{\mu}{ }^{\nu}\left(x, x^{\prime}\right) J_{\nu}\left(x^{\prime}\right)
$$

The equations of motion (3) imply that $G_{\mu}{ }^{\nu}$ satisfies the equation

$$
e_{\mu}{ }^{\nu \lambda} \partial_{\nu} G_{\lambda}{ }^{\alpha}+\mu G_{\mu}{ }^{\alpha}=-\frac{1}{\sqrt{-g}} g_{\mu}{ }^{\alpha} \delta\left(x-x^{\prime}\right),
$$

where $e_{\mu}{ }^{\nu \lambda}=g_{\mu \beta} \epsilon^{\beta \nu \lambda} / \sqrt{-g}$. In terms of vacuum expectation values of fields

$$
G_{\mu \nu}\left(x, x^{\prime}\right)=i\left\langle F_{\mu}(x) A_{\nu}\left(x^{\prime}\right)\right\rangle,
$$

and so

$$
\left\langle t^{\alpha \beta}\right\rangle=\lim _{x^{\prime} \rightarrow x} \frac{1}{i}\left(\epsilon^{\beta \gamma \sigma} \partial_{\gamma}^{\prime} G_{\sigma}^{\alpha}-\frac{1}{2} g^{\alpha \beta} g_{\mu \nu} \epsilon^{\nu \gamma \sigma} \partial_{\gamma}^{\prime} G_{\sigma}^{\mu}\right),
$$

where the limit $x^{\prime} \rightarrow x$ is to be taken symmetrically.

\section{Perfectly Conducting Parallel Lines}

For the case of parallel conducting lines, we can introduce a transverse spatial Fourier transform together with a Fourier transform in time:

$$
G^{\mu \nu}\left(x, x^{\prime}\right)=\int \frac{d \omega}{2 \pi} e^{-i \omega\left(t-t^{\prime}\right)} \int \frac{d k}{2 \pi} e^{i k\left(y-y^{\prime}\right)} \mathcal{G}^{\mu \nu}\left(k, \omega ; x, x^{\prime}\right) .
$$

It is straightforward to solve for the components $\mathcal{G}^{\mu \nu}$. The details are given in Ref. [1]. The result for the stress tensor on the (inside of) the bounding surfaces is

$$
t^{11}(0 \text { or } a)=\frac{i \kappa}{2} \cot \kappa a
$$

where $\kappa^{2}=\omega^{2}-k^{2}-\mu^{2}$. To find the force per unit length, we compute the discontinuity of $t^{11}$ across the boundary, and integrate over transverse momentum and frequency, after performing a Euclidean rotation:

$$
f=-\frac{1}{16 \pi a^{3}} \int_{2 \mu a}^{\infty} d y \frac{y^{2}}{e^{y}-1} \rightarrow-\frac{\zeta(3)}{8 \pi a^{3}}, \quad \text { as } \mu a \rightarrow 0 .
$$


This result, and the corresponding non-zero temperature result, are exactly the same as for a scalar field in the same geometry.

\section{Circular Boundary Conditions}

In this case we introduce the Fourier transform appropriate to the polar coordinates:

$$
G_{\mu}{ }^{\nu}\left(x, x^{\prime}\right)=\int \frac{d \omega}{2 \pi} e^{-i \omega\left(t-t^{\prime}\right)} \sum_{m=-\infty}^{\infty} e^{i m\left(\theta-\theta^{\prime}\right)} \mathcal{G}_{\mu}{ }^{\nu}\left(r, r^{\prime}\right) .
$$

Here, the calculation is considerably more involved, so we will content ourselves with presenting numerical results at $\mu=0$ and $T=0$ : The total Casimir force on the circle can be broken into two pieces, coming from $m=0$ and $m \neq 0$ terms: $F=F_{0}+F_{\mathrm{LT}}$, where

$$
F_{0}=-\frac{1}{2 \pi a^{2}} \int_{0}^{\infty} d x x \frac{d}{d x} \ln \left[2 x I_{1}(x) K_{1}(x)\right]=-\frac{0.254}{a^{2}}
$$

and

$$
F_{\mathrm{LT}}=-\frac{1}{4 \pi^{2} a^{2}} \int_{0}^{\infty} \frac{d y y}{e^{y}-1}=-\frac{1}{24 a^{2}} .
$$

The corresponding scalar result is six times smaller and opposite in sign. The force falls off rather rapidly with $\mu a$, being only $1 / 2$ the maximum value at $\mu a=2$. For a discussion of this and the temperature dependence, the reader is referred to Ref. [2].

\section{Discussion}

To get an idea of the magnitude of this effect, consider a circle of radius 100 $\AA$. According to (14), the corresponding Casimir energy is only about $5 \mathrm{eV}$. One could imagine a device consisting of a great many circular regions, so the coherent effect could be much larger. A much larger effect occurs for parallel lines as well: for two lines separated by $100 \AA$, the energy/length is about $4 \times 10^{5} \mathrm{eV} / \mathrm{cm}$. One must further bear in mind that the Chern-Simons field describing anyons is topological, and should not be confused with a observable Maxwell field; our speculation is that in two-dimensional confined geometries, the photon may acquire a topological mass, since such is not prohibited by gauge invariance. It remains a challenge to the experimenter to find a signature of this effect. We hope we have sown seeds of inspiration.

\section{Acknowledgements}

We thank the US Department of Energy for partial financial support.

\section{References}

1. K. A. Milton and Y. J. Ng, Phys. Rev. D 42 (1990) 2875.

2. K. A. Milton and Y. J. Ng, Phys. Rev. D 46 (1992) 842. 
